\title{
Évolution des proportions d'aubier et de duramen du cèdre de l'Atlas, Cedrus atlantica Manetti, en Algérie
}

Khellaf RABHI ${ }^{1}$

Mourad TAFER ${ }^{2}$

Mahand Messaoudene ${ }^{3}$

1 Université Hadj Lakhdar de Batna

Département d'agronomie 5 avenue Chahid Boukhlouf 05000 Batna

Algérie

2 Université Akli Mohand Oulhadj de Bouira Département d'agronomie Rue Drissi Yahia 10000 Bouira Algérie

${ }^{3}$ Institut national de recherche forestière Station régionale de Tizi Ouzou BP 30, Yakouren 15365 Tizi Ouzou Algérie
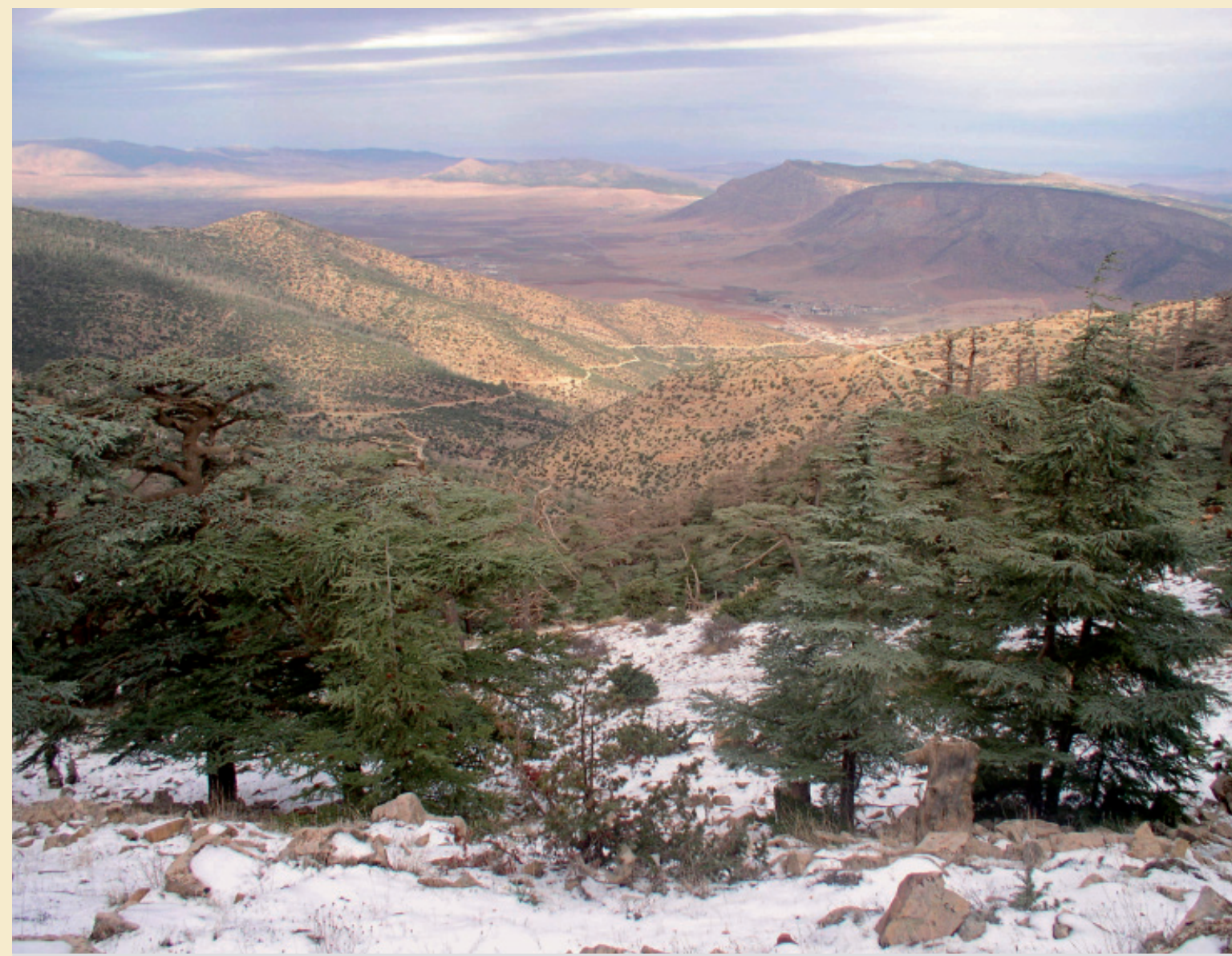

Photo 1.

Vue de la cédraie méridionale du Bélezma (Aurès) en contact avec l'Atlas saharien. Photo M. Messaoudene, 2012. 


\section{RÉSUMÉ}

\section{ÉVOLUTION DES PROPORTIONS D'AUBIER ET DE DURAMEN DU CÈDRE DE L'ATLAS, CEDRUS ATLANTICA MANETTI, EN ALGÉRIE}

L'étude a été conduite dans les cédraies du Bélezma (Aurès), du Djurdjura (Kabylie) et de Theniet El Had (Oranie), contrastées du point de vue bioclimatique et altitudinal. Elle s'inscrit dans le cadre global du projet sur le fonctionnement des cédraies algériennes. La problématique de l'étude est liée à l'absence de données sur le processus de duraminisation chez le cèdre de l'Atlas, données pourtant très utiles pour approfondir les connaissances sur la typologie des peuplements de cèdre. L'objectif était de mettre en évidence, dans un premier temps, l'évolution spatio-temporelle de la duraminisation. Sur l'ensemble des trois cédraies, 485 rondelles et carottes de bois ont été analysées. Les résultats montrent la faible variation des proportions du duramen d'une station à l'autre au sein d'une même cédraie. Globalement, l'âge affecte positivement la formation du duramen et négativement la proportion l'aubier. L'initiation de la duraminisation apparaît plus précoce dans les cédraies de Theniet El Had et Djurdjura (14 à 17 ans) et tardive dans les Aurès (20 à 30 ans). Dans cette dernière localité, le cèdre se singularise par une faible proportion d'aubier, variant de $45 \%$ à $50 \%$. Dans tous les cas, la cédraie du Djurdjura, située dans le bioclimat le plus humide, se distingue des deux autres cédraies par ses faibles proportions de duramen et fortes proportions d'aubier, respectivement $44 \%$ et $56 \%$. Le classement établi pour les proportions maximales d'aubier met au premier rang la cédraie de Talla Guillef dont la proportion d'aubier est de $78 \%$ et en dernier la cédraie du Bélezma avec un taux moyen de $68 \%$. Il se dégage de ce fait une tendance à l'évolution des proportions d'aubier en fonction du gradient bioclimatique en allant du perhumide au semi-aride. En outre, l'étude met en évidence la très faible proportion de l'aubier chez les arbres dépérissants, avec une valeur moyenne de $46 \%$.

Mots-clés : aubier, duramen, âge, cèdre de l'Atlas, Algérie.

\section{ABSTRACT}

\section{RESUMEN}

\section{VARIATION IN THE PROPORTIONS OF SAPWOOD AND HEARTWOOD IN ATLAS CEDARS, CEDRUS ATLANTICA MANETTI, IN ALGERIA}

This study was conducted in the cedar forests of the Belezma (Aurès), Djurdjura (Kabylia) and Theniet El Had (Orania) ranges, which vary widely in altitude as well as bioclimatically. The study was undertaken as part of an overall project on the functioning of Algeria's cedar forests. The problem addressed was the lack of data on the process of heartwood formation in Atlas cedars, which provides very useful information to improve knowledge on the typology of cedar forests. The aim was firstly to identify changes in heartwood formation over space and time. A total of 485 tree sections and cores from the three cedar forests were analysed. The results shows little variation in the proportions of heartwood from different stations in the same forest. Overall, age has a positive influence on heartwood formation and a negative influence on the proportion of sapwood. Heartwood formation appears to begin earlier in the Theniet El Had and Djurdjura forests (at 14 to 17 years of age) and later in the Aurès forests (at 20 to 30 years). Cedar trees in the latter characteristically have a lower proportion of sapwood, ranging from $45 \%$ to $50 \%$. In every case, the Djurdjura forest, which has the most humid bioclimate, differs from the two others in having a lower proportion of heartwood (44\%) and a higher proportion of sapwood (55\%). The highest proportion of sapwood, $78 \%$, was found in the Talla Guillef cedar forest, and the lowest in the Belezma forest, where the average was $68 \%$. This suggests a tendency for sapwood proportions to vary in accordance with a per-humid to semi-arid bioclimatic gradient. Our study also highlighted the very low proportion of sapwood in trees affected by dieback, where the average proportion was $46 \%$.

Keywords: sapwood, heartwood, age, Atlas cedar, Algeria.

\section{EVOLUCIÓN DE LA PROPORCIÓN DE ALBURA Y DURAMEN DEL CEDRO DEL ATLAS, CEDRUS ATLANTICA MANETTI, EN ARGELIA}

El estudio se realizó en los cedrales de Belezma (Aurés), Djurdjura (Cabilia) y de Theniet El Had (Región de Orán), que presentan contrastes bioclimáticos y altitudinales. Este trabajo se encuadra en el marco global del proyecto sobre el funcionamiento de los cedrales argelinos. La principal limitación del estudio viene dada por la ausencia de datos sobre el proceso de duraminización en el cedro del Atlas, datos que, sin embargo, son muy útiles para profundizar en el conocimiento sobre la tipología de los rodales de cedros. El propósito del estudio consistía, en un primer momento, en caracterizar la evolución espaciotemporal de la duraminización. Se analizaron 485 rodajas y cilindros de madera de los tres cedrales. Los resultados muestran la baja variación de las proporciones de duramen entre las distintas estaciones dentro de un mismo cedral. Globalmente, la edad afecta positivamente a la formación de duramen y negativamente a la proporción de albura. El inicio de la duraminización es más precoz en los cedrales de Theniet El Had y Djurdjura (14 a 17 años) y más tardía en el Aurés (20 a 30 años). En este último lugar, el cedro se caracteriza por una baja proporción de albura, que oscila entre $45 \%$ y $50 \%$. En todos los casos, el cedral de Djurdjura, ubicado en el bioclima más húmedo, se diferencia de los otros dos por su baja proporción de duramen y alta proporción de albura: $44 \%$ y $56 \%$, respectivamente. La clasificación en función de la mayor proporción de albura sitúa en primer lugar al cedral de Talla Guillef con un $78 \%$ y en el último lugar el cedral de Belezma con una tasa promedio del $68 \%$. De este hecho se desprende una tendencia a la evolución de la proporción de albura según el gradiente bioclimático que va del perhúmedo al semiárido. Además, el estudio pone de manifiesto la escasa proporción de albura en los árboles moribundos, con un valor promedio del $46 \%$.

Palabras clave: albura, duramen, edad, cedro del Atlas, Argelia. 


\section{Introduction}

La transformation de l'aubier en bois de cœur s'accompagne de modifications chimiques et physiques affectant la qualité de bois et conditionnant souvent ses possibilités d'emploi. Ce processus général peut être rattaché au phénomène naturel de vieillissement, d'accumulation d'air dans les tissus cellulaires devenus clos, puis de dévitalisation du parenchyme par des micro-organismes ou de mortalité sous l'effet d'une accumulation de polyphénols atteignant un seuil de toxicité (Polge, 1982). Taylor et al. (2002) estiment que la formation du duramen s'accompagne d'un changement de l'activité enzymatique, de la formation de produits d'extraction et de la mort des cellules du parenchyme, dont résulte la transformation de l'aubier en duramen.

Il existe le plus souvent une corrélation étroite entre le diamètre des arbres et la largeur de leur aubier, ce dont il peut être déduit que la duraminisation est un processus régulateur destiné à limiter la surface relative de l'aubier pour les besoins de conduction de la sève brute (Bamber, 1976). La connaissance de l'évolution spatio-temporelle de l'aubier permet d'appréhender la vitalité et la capacité de stockage en eau des arbres (Longuetaud, 2005). Par exemple, les épicéas présentent des surfaces d'aubier beaucoup plus faibles lorsqu'ils sont soumis à une défoliation (Longuetaud, 2005). Granier (1981), Jeffrey et Lachenbruch (2009) ont montré que la largeur d'aubier chez de nombreuses espèces était proportionnelle à la surface foliaire ou bien à la masse foliaire. En sylviculture, l'aubier est considéré comme un excellent indicateur de la qualité de la station, de la vigueur de la végétation et du patrimoine héréditaire (Polge, 1964). À ce titre, la densité par hectare des arbres d'une forêt est négativement corrélée avec les proportions respectives du duramen et de l'aubier (Jeffrey et Lachenbruch, 2009). S'agissant enfin de l'influence des conditions environnementales, l'aubier s'avère être lié davantage aux précipitations annuelles, contrairement au duramen (Climent et al., 1993). De l'avis de ces mêmes auteurs, les proportions de ces deux types de bois sont dépendantes de l'état du fonctionnement physiologique des arbres en relation avec les conditions stationnelles.

Dans cette étude, le fonctionnement spatio-temporel de l'aubier et du duramen du cèdre de l'Atlas, Cedrus atlantica Manetti, a été étudié dans trois cédraies algériennes contrastées du point de vue bioclimatique, édaphique et topographique : Theniet El Had (Tissemsilt), Djurdjura (Tizi Ouzou et Bouira) et Bélezma (Batna). Ce travail s'inscrit dans le contexte global d'un programme de recherche sur le fonctionnement écophysiologique du cèdre, en relation avec l'âge et les divers descripteurs du milieu.

\section{Matériel et méthodes}

Le matériel végétal étudié a été prélevé dans les cédraies du Bélezma, du Djurdjura et de Theniet El Had (figure 1).

La cédraie du Bélezma se situe dans la partie orientale de l'Algérie, près de la ville de Batna, et fait partie du grand massif montagneux des Aurès et du parc national éponyme. Son confinement dans la zone méridionale de hautes montagnes continentales, sise à la limite des grandes influences méditerranéennes et sahariennes, détermine sa particularité (photo 1). Son altitude varie de 1300 à $2180 \mathrm{~m}$, et la cédraie pure et mixte (cèdre et chêne vert) couvre une superficie de 5680 hectares. Du point de vue pédologique, Abdessemed (1981) relève la présence de sols bruns calcaires et de rendzines dolomitiques. Les deux zones de Boumerzoug et Touguert se caractérisent par des sols bruns calcaires faiblement profonds et squelettiques. Globalement, le Bélezma est confiné dans le bioclimat semi-aride à hiver froid.

La cédraie du parc national du Djurdjura chevauche les départements de Tizi Ouzou et de Bouira. C'est une zone de montagnes très escarpée, constituée de plusieurs chaînes calcaires composées de terrains sédimentaires fortement plissés et fracturés. Les sols sont de deux types, calcimagnésiques peu évolués et bruns forestiers acides. Le Djurdjura renferme deux cédraies bien venantes culminant à 1450 m d'altitude : Tikjda en exposition sud (photo 2) et Tala Guillef en exposition nord (photo 3). Ces deux cédraies n'ont pas été soumises au dépérissement ayant affecté celles du Bélezma et de Theniet El Had. Cette zone est située dans l'une des régions les plus arrosées et neigeuses d'Algérie, où la tranche pluviométrique annuelle est de l'ordre de 1200 à 1500 mm. Les deux cédraies sont respectivement dans les bioclimats perhumide et humide supérieur à hiver frais (Derridj, 1990).

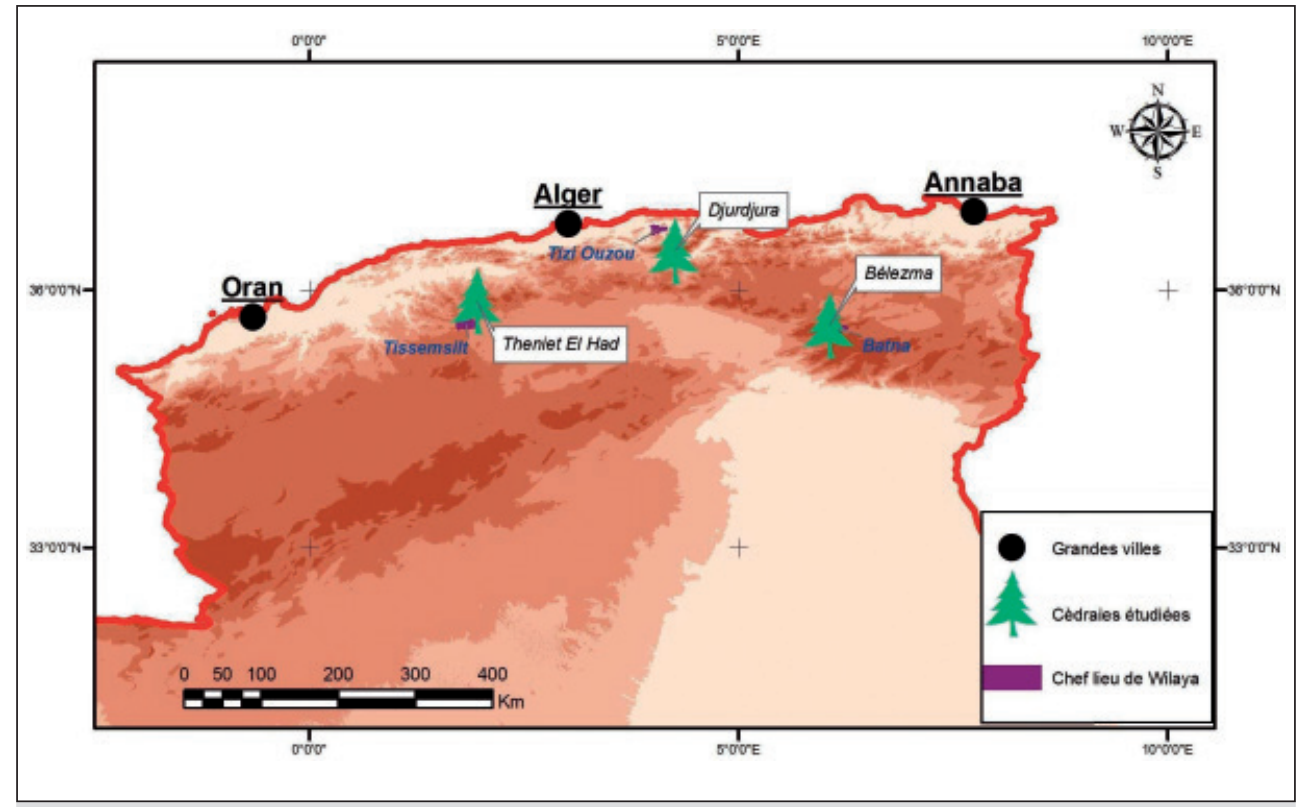

Figure1.

Situation géographique des trois cédraies étudiées en Algérie. Carte C. Chafai, 2013. 


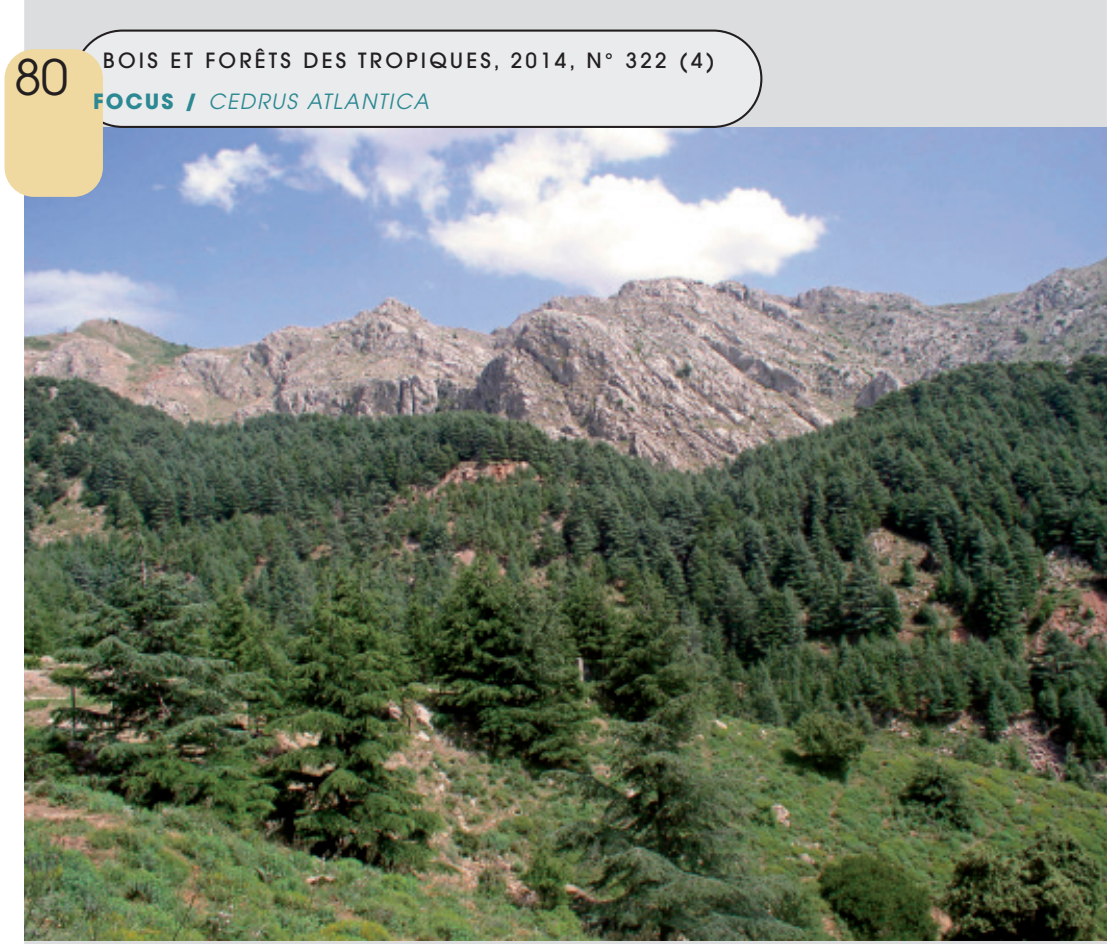

Photo 2.

Vue de la cédraie de Tikjda sur le versant sud du Djurdjura. Photo M. Messaoudene, 2012.

La cédraie de Theniet El Had (TEH) est située sur le versant sud de l'Atlas tellien et distante d'environ $50 \mathrm{~km} \mathrm{du}$ chef-lieu du département de Tissemsilt. Elle représente l'unique cédraie occidentale d'Algérie. Les peuplements de cèdre de l'Atlas apparaissent à partir de 862 m d'altitude, pour atteindre le point culminant de $1787 \mathrm{~m}$. Les sols sont peu évolués, d'apport colluvial, non carbonatés, peu profonds, jalonnés souvent par la roche mère et entrecoupés d'escarpements rocheux. Cette cédraie appartient à l'étage bioclimatique subhumide à hiver froid, où la saison sèche s'étale du mois de mai jusqu'au mois de septembre. Les précipitations annuelles sont irrégulières et s'élèvent en moyenne à 792 mm/an (DGF, 2006).
Dans le Bélezma, deux zones sont concernées par l'échantillonnage, le Djebel Boumerzoug et le Djebel Touguert. Selon quelques critères de milieu (la profondeur du sol, la pente, l'altitude et l'exposition), trois stations ont été décrites à Boumerzoug (B1, B2 et B3) et deux à Touguert (T1 et T2). Au Djurdjura, les sondages ont été réalisés à Tikjda (Tik) et à Tala Guillef (TG). À Theniet El Had (TEH), seulement une zone a été choisie. Dans la perspective d'appréhender l'impact de l'effet de l'état sanitaire des arbres sur la duraminisation, une centaine d'arbres sains ( $\mathrm{Sa}$ ) et dépérissants (Dp) ont été sondés dans la cédraie du Bélezma. Les stations ont été décrites en détail suivant sept critères : le nombre d'arbres $(\mathrm{N})$; l'âge moyen ; le diamètre moyen ; la pente (p) ; l'altitude ; la profondeur du sol (P.sol); le bioclimat (tableau I).

Dans cette étude, la pente moyenne et la profondeur du sol ont été estimées pour chaque station. Ainsi, la pente a été classée faible si $p<12 \%$, moyenne pour $12 \%<p<25 \%$, et forte si $p>25 \%$. La profondeur du sol a été subdivisée en trois niveaux : faible (1), moyen (2) et élevé (3).

En raison de l'importance du nombre d'échantillons et du statut de protection du cèdre de l'Atlas, une méthode non destructive a été appliquée, basée sur des prélèvements de carottes de $5 \mathrm{~mm}$ de diamètre assurés à l'aide d'une tarière de Presler. En revanche, sur les souches issues des coupes d'assainissement, des rondelles ont été prélevées. Contrairement à d'autres espèces où la ligne de séparation du duramen et de l'aubier est difficile à circonscrire en raison de la présence d'une zone de transition, chez le cèdre de l'Atlas, elle est plus facile à distinguer. De fait, l'approche de De Kort (1993), consistant à délimiter l'aubier et le duramen visuellement, a été mise en œuvre.

Tableau I.

Description des stations échantillonnées dans les trois cédraies.

\begin{tabular}{|c|c|c|c|c|c|c|c|c|c|c|}
\hline \multirow[b]{2}{*}{ Stations } & \multirow{2}{*}{\multicolumn{3}{|c|}{$\begin{array}{l}\text { Bélezma } \\
\text { Djebel Boumerzoug }\end{array}$}} & \multirow{2}{*}{\multicolumn{2}{|c|}{ Djebel Touguert }} & \multicolumn{2}{|c|}{ Djurdjura } & \multirow{2}{*}{$\begin{array}{c}\text { Theniet } \\
\text { El Had } \\
\text { TEH }\end{array}$} & \multicolumn{2}{|c|}{ État sanitaire } \\
\hline & & & & & & Tik & TG & & Sa & Dp \\
\hline Nombre d'arbres (N) & 70 & 120 & 67 & 64 & 103 & 53 & 78 & 30 & 35 & 65 \\
\hline Âge moyen (annèes) & 115,37 & 126,67 & 140,07 & 111,52 & 103,41 & 65,81 & 74,30 & 106,76 & 78,42 & 96,56 \\
\hline Diamètre moyen $(\mathrm{cm})$ & 17,08 & 20,90 & 26,86 & 35,22 & 24,12 & 35,20 & 31,62 & 37,88 & 25,94 & 24,58 \\
\hline Pente (p) & Faible & Moyenne & Forte & Faible & Forte & Forte & Forte & Moyenne & Moyenne & Forte \\
\hline Altitude (m) & 1400 & 1400 & 1470 & 1400 & 1550 & 1450 & 1450 & 1050 & 1400 & 1470 \\
\hline Profondeur du sol (m) & 1 & 1 & 1 & 2 & 1 & 3 & 3 & 2 & 1 & 1 \\
\hline Bioclimat & & & Semi-aride & & & $\mathrm{H}$ & $\mathrm{PH}$ & SH & & \\
\hline
\end{tabular}




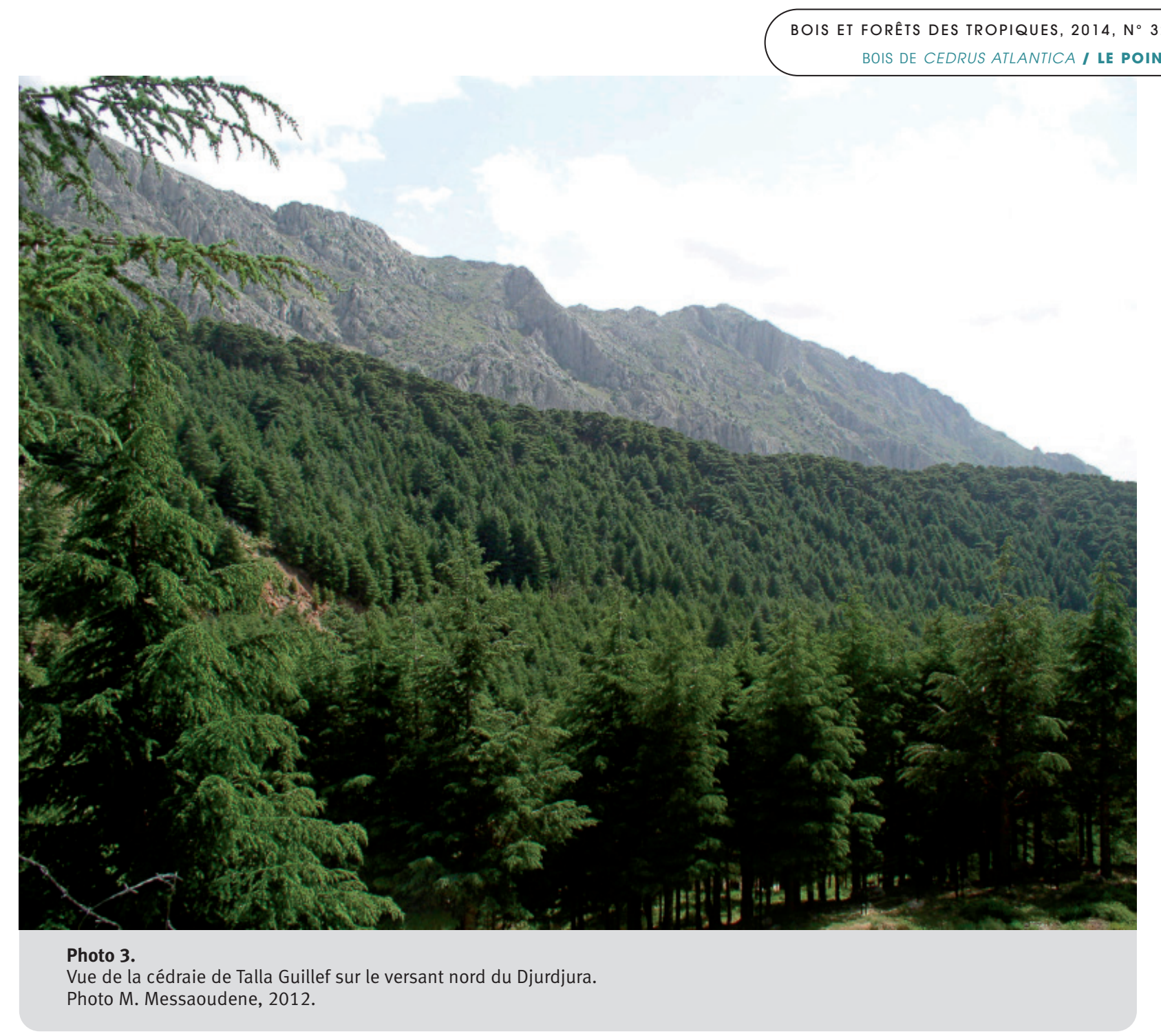

Après ponçage, polissage et préparation des carottes et des rondelles, les paramètres mesurés ont été les suivants : - AD, âge du duramen (nombre de cernes) ;

- AA, âge de l'aubier (nombre de cernes) ;

- AT, âge total ;

- SD, surface du duramen $\left(\mathrm{cm}^{2}\right)$;

- SA, surface de l'aubier $\left(\mathrm{cm}^{2}\right)$;

- RT, rayon total sous écorce de la carotte ou de la souche $(\mathrm{cm})$;

- \% SD, proportion en surface de duramen ;

- \% SA, proportion en surface d'aubier.

Les proportions surfaciques du duramen et de l'aubier ont été exprimées en pourcentage de la surface totale sous écorce. L'évolution de la proportion de l'aubier avec l'âge a été modélisée selon des régressions linéaires de forme générale : $\% \mathrm{SA}=\mathrm{a} \mathrm{AT}+\mathrm{b}$, où $\mathrm{a}$ et $\mathrm{b}$ sont des constantes à estimer.

Trois corrélations ont été étudiées :

- surface du duramen - surface terrière (SD-G) ;

- surface d'aubier - surface terrière (SA-G) ;

- proportion de la surface d'aubier - croissance moyenne (\% SA-Cr. moy.).

La détermination de l'âge à partir duquel le premier cerne se duraminise a été assurée à l'aide de régressions faisant intervenir le nombre de cernes du duramen (AD) comme facteur expliqué, et l'âge total comme facteur explicatif. Le point d'intersection des deux axes représente l'âge d'initiation de la duraminisation. D'après la répartition et la tendance affichée par le nuage de points, la forme linéaire de forme générale $[y=a \cdot x+b]$ a été retenue. Des modèles polynomiaux d'ordre 2 de forme $\left[y=a \cdot x^{2}+b \cdot x+c\right]$ peuvent aussi être utilisés. Mais bien qu'ils aient montré un bon ajustement pour plusieurs exemples traités dans la littérature (Longuetaud, 2005), ils ne permettent pas toujours d'interpréter l'intersection de la courbe avec l'axe des abscisses comme étant l'âge d'initiation du duramen, l'ajustement aux données conduisant parfois à un âge d'initiation négatif.

Dans les régressions $A D=f(A T)$, la pente de chaque modèle linéaire, estimée en nombre de cernes duraminisés annuellement, représente l'expansion du duramen ou la fraction de l'aubier qui se transforme en duramen annuellement. La variation de la vitesse d'expansion du duramen, suivant l'âge de l'arbre, est étudiée par l'estimation de la vitesse de duraminisation ( $\mathrm{mm} / \mathrm{an})$ par intervalles de 20,30 et 40 ans, choisis en fonction de la disponibilité des échantillons.

Le traitement des données a été axé sur des analyses descriptives, des corrélations ainsi que des régressions réalisées avec le logiciel Statistica. 


\section{Résultats et discussion}

\section{Proportions d'aubier et de duramen}

Les proportions des deux types de bois affichent des valeurs proches, les différences ne dépassant pas $6 \%$. À l'intérieur de la même cédraie, les proportions de duramen (\% SD) varient faiblement d'une station à l'autre. Elles oscillent de 50,11 \% à 55,34 \% au Bélezma (Aurès), de 43,47\% à 45,27 \% au Djurdjura, et atteignent 48,43\% à Theniet El Had. Le groupe d'arbres dépérissants et la station T1 présentent des proportions relativement plus élevées (\% SD = 54,11 et 55,34 ), mais la cédraie du Djurdjura détient les plus faibles proportions ( $\%$ SD $=43,47$ et 45,27 ) (tableau II, figures $2 a, 2 b$ et $2 c)$.

Contrairement au duramen, les proportions d'aubier enregistrées dans les deux cédraies du Djurdjura sont les plus élevées (\% SA $=54,72$ et 56,52 ) ; vient ensuite celle de Theniet El Had (\% SA = 51,56), puis celles du Bélezma (\%SA $=51,13$ et 45,88$)$. Ce classement par rapport aux proportions maximales de l'aubier, bois fonctionnel, semble caractériser davantage les cédraies situées dans le bioclimat très humide, ce qui est le cas de celle de Tala Guillef, située dans le perhumide et exposée au Nord. Il apparaît donc une tendance d'évolution de l'aubier en fonction d'un gradient bioclimatique allant du perhumide au semi-aride, voire un effet régional sur la quantité d'aubier. De fait, la cédraie du Djurdjura semble présenter les conditions de croissance les plus favorables pour la formation d'un épais aubier et être plus fertile (Bamber, 1987 ; Pinto et al., 2004). Dans ce sens, Polge (1964) note que la proportion d'aubier est un bon indicateur de la fertilité de la station, tandis que Climent et al. (1993) considèrent qu'elle est beaucoup plus liée aux précipitations annuelles.

Bien qu'il soit démontré que la proportion d'aubier diminue avec l'âge (De Kort, 1993), les résultats obtenus ne peuvent être rattachés à ce facteur, d'autant plus que l'âge moyen de la cédraie de Tala Guillef (74 ans) est supérieur à celui de Tikjda (66 ans), alors que ceux de Theniet El Had et Bélezma sont très proches (106 et 119 ans).

\section{Figure 2.}

Présentation schématique des âges (total, du duramen et de l'aubier) des échantillons (a), des surfaces (total, du duramen et de l'aubier) des échantillons (b), des proportions en surface du duramen et de l'aubier (c).

$A D$ : âge du duramen (nombre de cernes); $A A$ : âge de

l'aubier (nombre de cernes) ; AT : âge total ; SD : surface de duramen $\left(\mathrm{cm}^{2}\right)$; SA : surface de l'aubier $\left(\mathrm{cm}^{2}\right)$; \% SD : proportion de duramen en surface ; \% SA : proportion d'aubier en surface; B1, B2 et B3 : stations de Bélezma dans le Djebel Boumerzoug ; T1 et T2 : stations de Bélezma dans le Djebel Touguert; Tik (Tikjda) et TG (Tala Guillef) : stations de Djurdjura ; TEH : station de Theniet El Had ; Sa (arbres sains) et Dp (arbres dépérissants) : état sanitaire ; ST : surface totale de la section ou surface terrière sous écorce.

Tableau II.

Présentation des résultats des mesures effectuées dans les trois cédraies.

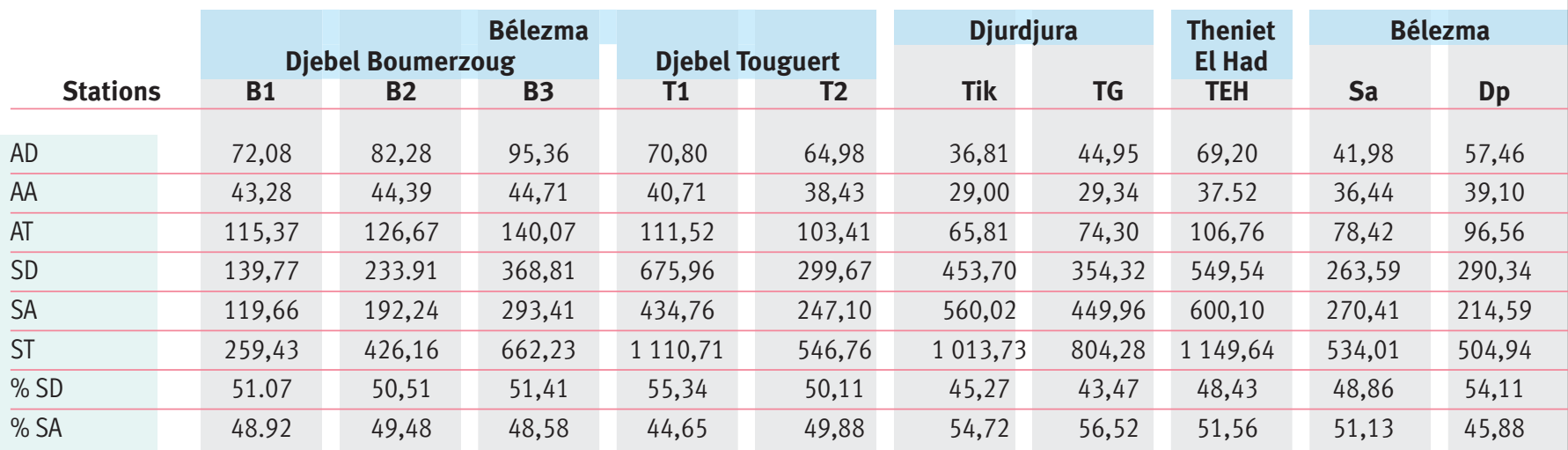

$A D$ : âge du duramen (nombre de cernes); $A A$ : âge de l'aubier (nombre de cernes) ; $A T$ : âge total ; SD : surface de duramen ( $\left.\mathrm{cm}^{2}\right)$; $S A$ : surface de l'aubier $\left(\mathrm{cm}^{2}\right) ; \mathrm{ST}=\mathrm{SD}+\mathrm{SA}$ : surface totale de la section à 1,3 $\mathrm{m} ; \% \mathrm{SD}$ : proportion de duramen en surface ; \% SA : proportion d'aubier en surface ; Tik : Tikjda ; TG : Tala Guillef ; TEH : Theniet El Had. 
Au Bélezma, la comparaison entre les arbres sains (Sa) et dépérissants (Dp) montre que les cèdres sains ont un aubier plus épais, avec des valeurs de \% A s'élevant respectivement à 51,13 et 45,88. Le test de Student pour comparer les deux échantillons a révélé que la différence était significative au seuil alpha de $\alpha=0,05(t=2,4920 ; P=0,0134)$. Ce résultat corrobore ceux d'auteurs notant que le mauvais état sanitaire des arbres réduit la proportion d'aubier. Hazenberg et Yang (1991) et Jeffrey et Lachenbruch (2009) mettent en évidence une relation de cause à effet entre l'aubier, d'une part, et la taille des couronnes et la biomasse foliaire fonctionnelle, d'autre part. De Kort (1993) déduit que la vitalité des arbres est dépendante de leur état sanitaire, et que les plus faibles valeurs de l'aubier correspondent aux arbres les moins vitaux. Ainsi, le dépérissement observé dans la cédraie du Bélezma, marqué par des pertes progressives de la biomasse foliaire et l'assèchement des branches, expliquerait cette tendance à la décroissance de l'aubier chez les arbres dépérissants (Dp).

Dans tous les cas, les conditions topographiques (altitude et pente) ne semblent pas influencer les proportions des deux types de bois (aubier et duramen). À ce stade de l'analyse et en s'appuyant sur le test de Student, les différences entre les stations du Bélezma ne sont pas significatives au seuil $\alpha=0,05$. Le même constat est noté pour l'influence éventuelle de la profondeur du sol.

\section{Évolution de la proportion de l'aubier avec l'âge}

Pour toutes les stations, les modèles linéaires ajustés entre la proportion d'aubier et l'âge montrent que la proportion d'aubier maximale (\% SA max.) observée sur les jeunes sujets varie de 61,20 \% (B2) à 91,90 \% (Dp) (tableau III). Les coefficients négatifs affichés par tous les modèles renseignent sur la tendance baissière qui caractérise la proportion d'aubier avec l'âge. En effet, une baisse annuelle très élevée est notée pour les arbres dépérissants (Dp). Elle est de 0,487 \% par an, alors que le rythme de diminution le plus faible est observé dans la station B2 (0,092\%/an). Dans la majorité des cédraies, les modèles linéaires déduits sont significatifs, à l'exception de ceux obtenus des stations B1, T2 et TEH.
On peut admettre que l'âge de l'arbre affecte positivement la proportion de duramen et négativement la proportion d'aubier. En effet, nous savons que les proportions d'aubier et de duramen dans un arbre dépendent de la vitesse de croissance et de celle de l'expansion du duramen. L'activité cambiale diminue avec le vieillissement de l'arbre, contrairement à la croissance du duramen, ce qui explique nos résultats. Dans la même optique, De Kort (1993) montre que les plus vieux arbres du sapin baumier ont le taux le plus faible d'aubier, contrairement aux plus jeunes où la proportion d'aubier est élevée.

Prenant en compte l'effet de l'âge sur ce processus, la production de bois riche en duramen tel que recherché pour la construction est favorisée par une croissance lente et une exploitation tardive. Cependant, la production de bois avec une faible proportion de duramen, comme il est préconisé pour les industries papetières, est favorisée par une croissance rapide et une récolte précoce. Les traitements sylvicoles ayant comme objectif l'accélération de la croissance radiale contribueraient à cet objectif. De ce point de vue, Jeffrey et Lachenbruch (2009) remarquent que les arbres dominants montrent un pourcentage élevé d'aubier et que l'élargissement des couronnes résultant notamment des éclaircies s'accompagne d'une augmentation de la proportion d'aubier. Polge (1985) observe que les arbres sévèrement élagués présentent une accélération de la duraminisation et un pourcentage plus élevé de bois de cœur. Ce résultat est expliqué par la théorie d'efficience de la duraminisation de Bamber (1976) puisque la diminution du volume du houppier par les élagages sévères entraîne des besoins moindres de circulation de sève, nécessitant donc des surfaces plus faibles d'aubier.

Des corrélations positives très fortes sont notées pour la relation rayon du duramen - rayon total (D-RT) (tableau IV). À part les arbres dépérissants (Dp), les corrélations positives observées entre la surface d'aubier (SA) et la surface terrière (G) sont hautement significatives, dépassant dans tous les cas 0,81 et atteignant 0,96 (tableau IV). Ce résultat indiquerait que les arbres ont besoin d'un taux particulier d'aubier pour assurer un parfait fonctionnement. Dans la mesure où la quantité d'aubier doit être continuellement accrue avec la crois-

\section{Tableau III.}

Les modèles de la proportion d'aubier en fonction de l'âge total : \% $S A=f(A T)$.

\begin{tabular}{|c|c|c|c|c|}
\hline Stations & Modèles ajustés & $\mathbf{R 2}$ & $\%$ SA max. & Diminution (\% A/an) \\
\hline B2 & $\% S A=-0,092 A T+61,20$ & 0,32 & 61,20 & 0,092 \\
\hline B3 & $\% \mathrm{SA}=-0,124 \mathrm{AT}+65,66$ & 0,23 & 65,66 & 0,124 \\
\hline $\mathrm{T} 1$ & $\% \mathrm{SA}=-0,300 \mathrm{AT}+77,06$ & 0,25 & 77,06 & 0,300 \\
\hline Tik & $\% \mathrm{SA}=-0,283 \mathrm{AT}+73,34$ & 0,38 & 73,34 & 0,283 \\
\hline$\overline{\mathrm{TG}}$ & $\% \mathrm{SA}=-0,292 \mathrm{AT}+78,23$ & 0,30 & 78,23 & 0,292 \\
\hline Sa & $\% \mathrm{SA}=-0,178 \mathrm{AT}+66,27$ & 0,43 & 66,27 & 0,178 \\
\hline$\overline{D p}$ & $\% \mathrm{SA}=-0,487 \mathrm{AT}+91,90$ & 0,46 & 91,90 & 0,487 \\
\hline
\end{tabular}

\% SA : proportion d'aubier en surface ; Tik : Tikjda ; TG : Tala Guillef ; Sa : arbres sains ; Dp : arbres dépérissants ; AT : âge total. 
sance de la couronne, l'augmentation de la biomasse foliaire agirait aussi positivement sur la croissance. Le taux optimum d'aubier est maintenu par la formation du duramen, celle-ci agissant alors comme un mécanisme de régulation pour contrôler la quantité d'aubier. L'efficience de cette régulation a été mise en évidence par Bamber (1976). L'auteur met ce processus régulateur en relation directe avec les dépenses relatives à l'alimentation des cellules vivantes de l'aubier excédentaire que l'arbre reconvertit en matériaux inertes, dont résulte la formation de duramen. Les corrélations significatives observées convergent vers la théorie de Björklund (1999) suggérant que le duramen est produit avec une fraction constante de l'accroissement annuel. Ces corrélations peuvent donc être attribuées au fait qu'une partie constante de l'accroissement annuel se transforme en duramen. Ces résultats sont confortés par les corrélations positives constatées entre la proportion en surface d'aubier (\% SA) et la croissance moyenne en millimètres par an. À l'exception de la station TEH pour laquelle nous avons noté une absence de corrélation linéaire $(r=0,08)$, les autres stations montrent des corrélations positives significatives (tableau IV), ce qui révèle que la croissance ralentit quand la proportion d'aubier diminue.

\section{L’âge d'initiation de la duraminisation}

Les résultats obtenus sont récapitulés dans le tableau $V$ et la figure 3. L'âge d'initiation de la duraminisation est plus précoce dans les cédraies de Theniet El Had et Tikjda où ce processus démarre approximativement dès l'âge de 14 ans. Au Djurdjura, un décalage de deux ans et demi s'observe entre les deux cédraies (Tikjda et Tala Guillef), le démarrage étant plus tardif à Tala Guillef (environ 17 ans). Au Bélezma, ce processus intervient à des âges compris entre 25 et 30 ans à Djebel Boumerzoug alors qu'il est moins tardif à Djebel Touguert (20 à 24 ans) (tableau V). À ce stade de l'analyse, l'effet de la station sur l'âge d'initiation de la duraminisation apparaît bien.

Dans tous les cas, un gradient de démarrage de la duraminisation se dessine et semble prendre en compte la vitalité des peuplements ainsi que la position bioclimatique des cédraies. Au Bélezma, la comparaison des données de Touguert (exposition Nord) et de Boumerzoug soumis aux influences sahariennes (exposition Sud) permet de conclure que les arbres évoluant dans des conditions de croissance difficiles tendent à retarder le démarrage de ce processus.

Tableau IV.

Présentation des résultats des corrélations.

\begin{tabular}{|l|c|c|c|c|c|c|c|c|c|c|} 
Paramètres & B1 & B2 & B3 & T1 & T2 & TEH & Tik & TG & Sa & Dp \\
\hline D-RT & 0,95 & 0,98 & 0,98 & 0,98 & 0,97 & 0,68 & 0,85 & 0,81 & 0,84 & 0,90 \\
\hline SA-G & 0,96 & 0,94 & 0,90 & 0,87 & 0,92 & 0,83 & 0,92 & 0,81 & 0,78 & 0.66 \\
\hline \% SA-Cr. moy. & 0,24 & 0,28 & 0,26 & 0,47 & 0,23 & 0,08 & 0,30 & 0,45 & 0,60 & 0,52 \\
\hline
\end{tabular}

$r$ critique $=0,22$ (valeur au-dessous de laquelle le coefficient de corrélation reste non significatif).

$D$ : rayon du duramen $(\mathrm{cm}) ; R T$ : rayon total $(\mathrm{cm}) ; S A$ : surface d'aubier $\left(\mathrm{cm}^{2}\right) ; G$ : surface terrière $\left(\mathrm{cm}^{2}\right) ; \%$ SA : proportion en surface de l'aubier ; Cr. moy. : croissance moyenne en mm/an ; TEH : Theniet El Had ; Tik : Tikjda ; TG : Tala Guillef ; Sa : arbres sains ; Dp : arbres dépérissants.

Tableau V.

Les modèles de l'âge du duramen en fonction de l'âge total : $A D=f(A T)$.

\begin{tabular}{|c|c|c|c|c|c|}
\hline & Stations & Relations ajustées & $r^{2}$ & $x: y=0$ & Taux dur. (mm/an) \\
\hline \multirow[t]{5}{*}{ Bélezma (Aurès) } & B1 & $A D=0,917 A T-23,21$ & 0,83 & 25,31 & 0,49 \\
\hline & B2 & $A D=0,818 A T-21,43$ & 0,95 & 26,19 & 0,53 \\
\hline & B3 & $A D=0,868 A T-26,03$ & 0,94 & 29,98 & 0,30 \\
\hline & $\mathrm{T} 1$ & $A D=0,796 A T-16,17$ & 0,94 & 20,31 & 1,19 \\
\hline & T2 & $A D=0,814 A T-19,22$ & 0,89 & 23,61 & 0,83 \\
\hline \multirow[t]{2}{*}{ Djurdjura } & Tik & $A D=0,713 A T-10,14$ & 0,68 & 14,22 & 1,82 \\
\hline & TG & $A D=0,687 A T-11,47$ & 0,93 & 16,69 & 1,43 \\
\hline Theniet El Had & TEH & $A D=0,754 A T-10,66$ & 0,77 & 14,13 & 1,40 \\
\hline \multirow[t]{2}{*}{ Bélezma } & Sa & $A D=0,735 A T-12,73$ & 0,91 & 17,32 & 1,29 \\
\hline & Dp & $A D=0,909 A T-30,35$ & 0,86 & 30,65 & 0,94 \\
\hline
\end{tabular}

AD : nombre de cernes du duramen ; Sa : arbres sains ; Dp : arbres dépérissants ; Tik : Tikjda ; TG : Tala Guillef ; TEH : Theniet El Had. 

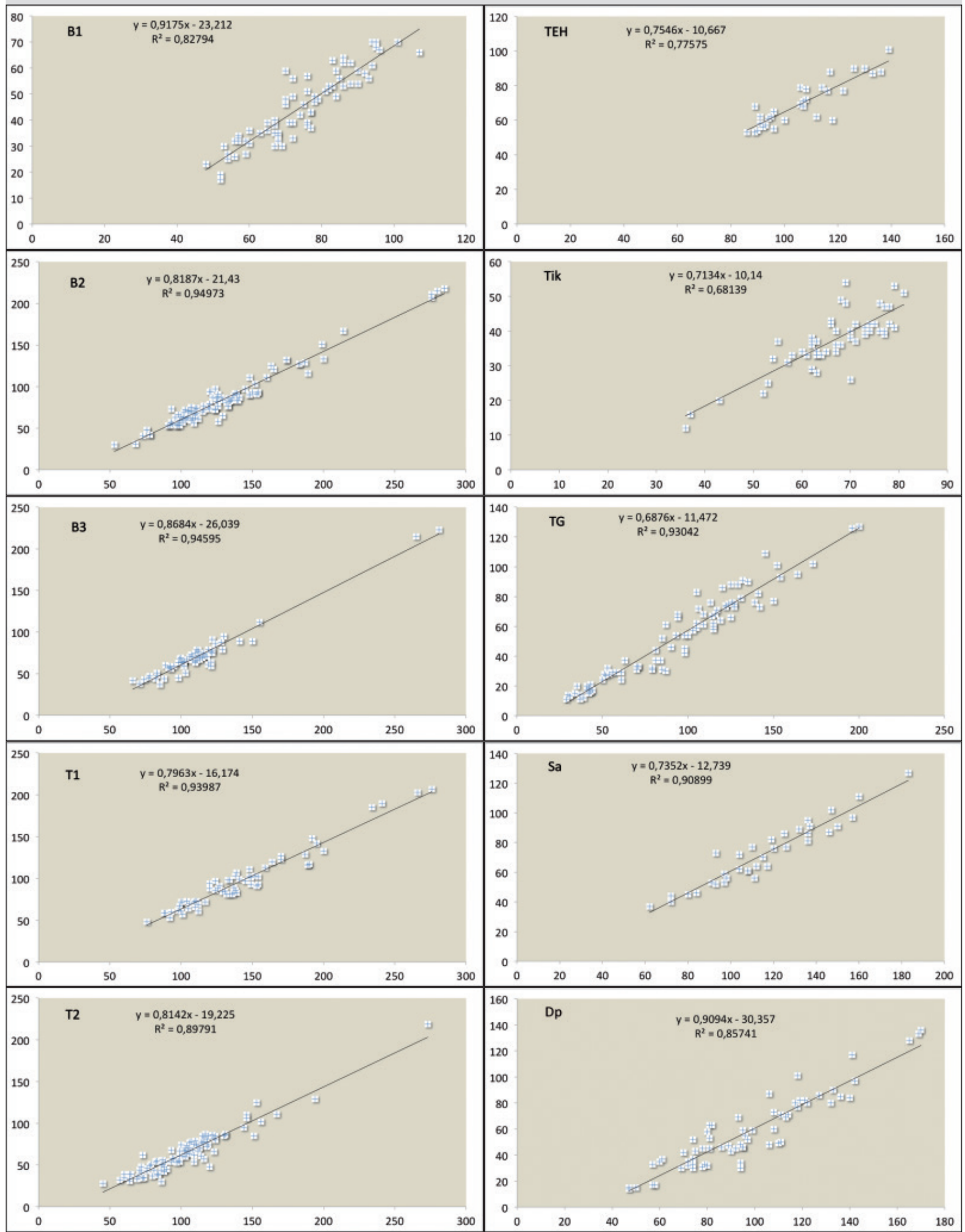

Figures 3.

Les modèles de l'âge du duramen (AD) en fonction de l'âge total (AT) : $A D=f(A T)$.

B1, B2 et B3 : stations de Bélezma dans le Djebel Boumerzoug ; T1 et T2 : stations de Bélezma dans le Djebel Touguert ; Tik (Tikjda) et TG (Tala Guillef) : stations de Djurdjura ; TEH : station de Theniet El $\mathrm{Had}$; Sa (arbres sains) et Dp (arbres dépérissants) : état sanitaire ; Dp : arbres dépérissants. 
Ce dernier démarre à un âge moyen d'environ 31 ans. La duraminisation est en outre plus précoce au sein des cédraies du Djurdjura évoluant dans le perhumide, et plutôt tardive dans les cédraies méridionales comme celle du Bélezma, en zone semi-aride. De Kort (1993) et Björklund (1999) concluent que chez la plupart des arbres forestiers, dans des conditions environnementales favorables, la duraminisation tend à démarrer à des âges très jeunes. Cependant, son initiation peut être différente en fonction de l'essence, du contraste du milieu et de la provenance. Par comparaison, elle se manifeste à 17 ans pour l'épicéa commun (Longuetaud, 2005), 41 ans pour le bouleau (Giraud, 2005) et 9 à 38 ans pour les pins (Hazenberg et Yang, 1991 ; Björklund, 1999 ; Pinto et al., 2004). Citant plusieurs travaux, Björklund (1999) confirme que l'âge d'initiation varie également au sein d'une même essence ; chez le pin sylvestre, par exemple, il est de 30-40 ans en Finlande et en Allemagne (Kuhn, 1918 ; Lappi-Seppala, 1952), mais s’abaisse à 25 ans en Suède (Fries et Ericsson, 1998).

\section{L'expansion du duramen}

Les résultats relatifs à la vitesse d'expansion du duramen, représentée par la pente de chaque modèle linéaire et estimée en nombre de cernes duraminisés par année, ne diffèrent pas grandement ; ils varient de 0,687 (TG) à 0,917 cerne par an (B1) (tableau V). Pour prendre en compte le rythme de croissance radiale qui diffère a priori d'une cédraie à une autre, la vitesse de duraminisation transformée en millimètres de rayon duraminisé par année montre que, globalement, les taux obtenus sont d'autant plus élevés que les arbres évoluent dans des conditions favorables. Ainsi, la cédraie de Tikjda se singularise et montre un rythme de duraminisation annuel plus important (1,82 mm/an) qu'à Tala Guillef et Theniet El Had. Le plus faible rythme de duraminisation est observé au niveau des stations du Bélezma et du groupe d'arbres dépérissants. Bamber (1987) suggère que la formation de duramen pour Pinus radiata est encouragée par des conditions hydriques favorables. Cette vitesse est comprise entre 0,5 et 1 cerne par an chez le genre Pinus (Hazenberg et Yang, 1991 ; Björklund,
1999 ; Pinto et al., 2004). Pour expliquer cette tendance à l'expansion du duramen, ces auteurs ont développé des modèles de régression reliant l'âge à la quantité de duramen.

Dans cette étude, les résultats montrent que la tendance de l'évolution est plus ou moins similaire entre les stations (figure 4). Les courbes présentent le même profil, ce qui signifie que le contraste écologique qui caractérise les dix échantillons n'affecte pas cette évolution. À l'exception du groupe d'arbres dépérissants (Dp) sur lequel nous observons une relative stabilité avec l'âge, les autres montrent une augmentation de la vitesse de duraminisation. En revanche, le groupe d'arbres sains (Sa) suit une certaine linéarité où le rythme d'augmentation est faible. Chez le sapin baumier, le duramen augmente à un taux moyen de 0,81 cerne par an de 10 à 90 ans, puis progresse ensuite pour atteindre un cerne par an. Cette augmentation avec l'âge est expliquée par les besoins de soutien mécanique de plus en plus élevés de l'arbre (Hazenberg et Yang, 1991).

La diminution de la vitesse annuelle de la croissance radiale, observée chez le cèdre sur de longues chronologies par plusieurs auteurs (Messaoudene et al., 2004 ; Sarmoum, 2008 ; Alilèche, 2011), laisse penser que le taux de duraminisation augmenterait avec une amplitude beaucoup plus importante s'il était exprimé en cernes par an. Cette constatation est confortée par les résultats de Polge (1982), qui a mis en évidence que pour les arbres fortement concurrencés, soumis à des compétitions très accrues, l'aubier, aussi mince soit-il, se maintient sur une période de temps relativement longue. Dans notre cas, la formation de cernes minces dans l'aubier résulterait du vieillissement des arbres.

\section{Conclusion}

Dans ce travail, le processus de duraminisation du bois de cèdre de l'Atlas d'Algérie a été étudié à l'échelle de trois cédraies contrastées du point de vue des conditions de milieu, notamment bioclimatiques. Globalement, l'effet station apparaît comme influençant les taux du duramen et d'aubier, mais l'évolution temporelle de la vitesse d'expan-

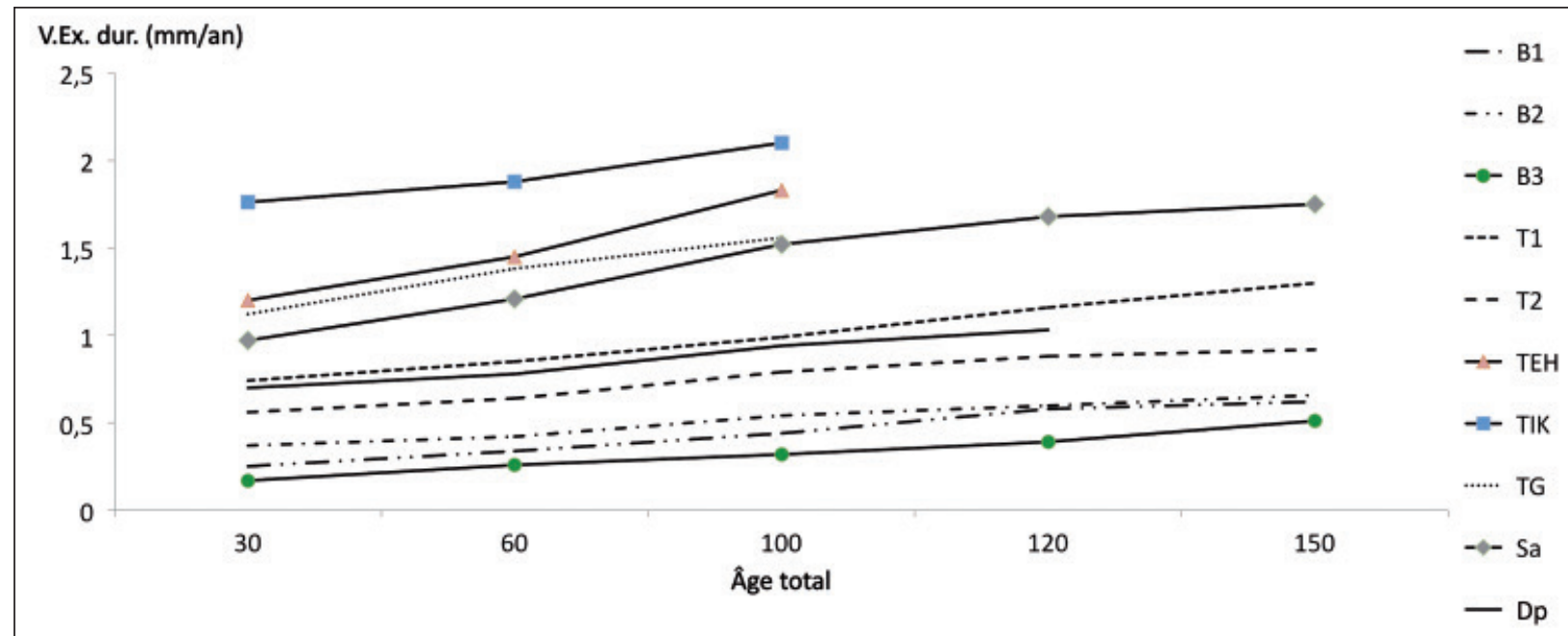

Figure 4.

Évolution de la vitesse de duraminisation du cèdre $(\mathrm{mm} / \mathrm{an})$ en fonction de l'âge.

B1, B2 et B3 : stations de Bélezma dans le Djebel Boumerzoug ; T1 et T2 : stations de Bélezma dans le Djebel Touguert ; Tik (Tikjda) et TG (Tala Guillef) : stations de Djurdjura ; TEH : station de Theniet El Had ; Sa (arbres sains) et Dp (arbres dépérissants) : état sanitaire ; 11. V.Ex. dur : vitesse d'expansion du duramen ; Dp : arbres dépérissants. 
sion du duramen n'est pas dépendante des conditions de milieu. En revanche, la part d'aubier transformée annuellement en duramen est étroitement liée aux conditions climatiques ; les taux de duraminisation les plus élevés caractérisent les cédraies situées dans les bioclimats perhumide et humide (Tikjda et Tala Guillef dans le Djurdjura). Quelle que soit la cédraie, en situation défavorable, l'arbre a tendance à contenir davantage de duramen, et donc une proportion faible d'aubier. L'état sanitaire de l'arbre, plus particulièrement l'état de son houppier, exerce un effet prépondérant sur la proportion d'aubier ; les arbres dépérissants présentent des proportions d'aubier très faibles.

L'âge d'initiation de la duraminisation est d'autant plus précoce que les arbres évoluent dans des conditions de plus en plus favorables; il est de fait tardif dans les cédraies relevant de zones semi-arides. La vitesse d'expansion du duramen augmente quant à elle avec l'âge. Les modèles linéaires décroissants ajustés attestent que la proportion d'aubier montre une tendance inverse, en diminuant avec l'âge.

La formation d'une forte proportion d'aubier chez le cèdre de l'Atlas, associée à un rythme rapide de la croissance radiale, permettrait l'obtention de bois à usage de papeterie, alors qu'une croissance lente et une exploitation tardive seraient plus favorables à la formation du duramen, utile à l'industrie de bois d'œuvre. Les quantités de ces deux types de bois peuvent être modulées en fonction des usages par des pratiques sylvicoles ; l'accélération de la croissance radiale résultant des éclaircies favoriserait la formation d'aubier.

En perspective, il est envisagé ultérieurement de s'intéresser à l'établissement d'une liaison entre la proportion d'aubier et la biomasse foliaire de chaque arbre pour vérifier l'application de la théorie du Pipe model, théorie selon laquelle le tronc et les branches d'une plante peuvent être considérés comme un ensemble de tuyaux dont chacun supporte une certaine quantité bien définie de feuilles. Par ailleurs, dans le contexte de l'évaluation de la qualité du bois de cèdre, notamment sa durabilité naturelle, l'étude de la dynamique spatio-temporelle des polyphénols dans le duramen et de l'aubier est envisagée de manière à caractériser typologiquement ces deux types de bois.

\section{Références bibliographiques}

ABDESSEMED K., 1981. Le Cèdre de l'Atlas dans les massifs de l'Aurès et du Bélezma. Étude phytosociologique. Problèmes de conservation et d'aménagement. Thèse de docteur-ingénieur, Faculté Saint-Jérôme, Université Aix-Marseille III, 199 p.

ALILÈCHE A., 2011. Étude du dépérissement du cèdre de l'Atlas dans les cédraies des Aurès (Bélezma et Chélia). Mémoire Magister, USTHB, Algérie, 151 p.

BAMBER R. K., 1976. Heartwood, its function and formation. Wood Science and Technology, 10 (1): 1-8.

BAMBER R. K., 1987. Sapwood and Heartwood. Beecroft, Australie, Forestry Commission of New South Wales, Wood Technology and Forest Research Division, Technical Publication $\mathrm{n}^{\circ} 2,8 \mathrm{p}$.

BJÖRKLUND L., 1999. Identifying heartwood-rich stands or stems of Pinus sylvestris by using inventory data. Silva Fennica, 33 (2): 119-129.
CLIMENT J., GIL L., PARDOS J., 1993. Heartwood and sapwood development and its relationship to growth and environment in Pinus canariensis Chr. Sm ex DC. Forest Ecology and Management, 59: 165-174.

DE KORT I., 1993. Relationships between sapwood amount, latewood percentage, moisture content and crown vitality of Douglas fir, Pseudotsuga menziensii. IAWA Journal, 14 (4): 413-427.

DERRIDJ A., 1990. Étude des populations de Cedrus atlantica M. en Algérie. Thèse, Université Paul Sabatier, Toulouse, France, $288 \mathrm{p}$.

DGF, 2006. Atlas des parcs nationaux. Direction générale des forêts, Algérie, 96 p.

FRIES A., ERICSSON T., 1998. Genetic parameters in diallelcrossed Scots pine favor heartwood formation breeding objectives. Canadian Journal of Forest Research, 28: 1-5.

GRANIER A., 1981. Étude des relations entre la section du bois d'aubier et la masse foliaire chez le Douglas (Pseudotsuga menziensii Mirb. Franco). Annals of Forest Science, 38 (4) : 503-512.

HAZENBERG G., YANG K. C., 1991. Sapwood/heartwood width relationships with tree age in balsam fir. IAWA Journal, 12 (1): 95-99.

JEFFREY D. D., LACHENBRUCH B., 2009. Heartwood/sapwood variation of western redcedar as influenced by cultural treatments and position in tree. Forest Ecology and Management, 258: 2026-2032.

KUHN W., 1918. Die Kiefernstarkholzsucht in einzel-, gruppen- und bestandweisen Überhalt. München.

LAPPI-SEPPÄLÄ M., 1952. Männyn sydänpuusta ja runkomuodosta. [On heartwood and stem form of Scots pine.] Communicationes Instituti Forestalis Fenniae 40(25). (In Finnish with German summary.)

LONGUETAUD F., 2005. Détection et analyse non destructive de caractéristiques internes de billons d'épicéa commun (Picea abies (L.) Karst.) par tomographie à rayons $\mathrm{X}$. Thèse, Engref, Nancy, France, $124 \mathrm{p}$.

MESSAOUDĖNE M., LOUKKAS A., JANIN G., TAFERE M., DILEM A., GONÇALEZ J., 2004. Propriétés physiques du bois d'éclaircie des cèdres (Cedrus atlantica), contenant du bois de compression, provenant de l'Atlas du Djurdjura (Algérie). Annals of Forest Science, 61 (6) : 589-595.

PINTO I., PEREIRA H., USENIUS A., 2004. Heartwood and sapwood development within maritime pine (Pinus pinaster Ait) stems. Trees, 18 (3): 284-294.

POLGE H., 1964. Délimitation de l'aubier et du bois de cœur par analyse densitométrique de clichés radiographiques. Annals of Forest Science, 21 (4) : 605-623.

POLGE H., 1982. Influence de la compétition et de la disponibilité en eau sur l'importance de l'aubier du douglas. Annals of Forest Science, 39 (4) : 379-398.

POLGE H., 1985. Influence de l'élagage sur la duraminisation, la production de bois de tension et quelques autres propriétés du bois de peuplier I 214. Annals of Forest Science, 42 (3) : 283-296.

SARMOUM M., 2008. Impact du climat sur le dépérissement du cèdre de l'Atlas (Cedrus atlantica Manetti). Diagnostic dendroécologique et climatique de la cédraie de Theniet El Had. Mémoire de Magister, USTHB, Alger, Algérie, 98 p.

TAYLOR A. M., GARTNER B. L., MORRELL J. J., 2002. Heartwood formation and natural durability - A review. Wood and Fiber Science, 34 (4): 587-611. 